\title{
petite fille blanche éprise de neige
}

\author{
jean-sébastien trudel
}

munste belongs to a tiny line of diverse characters such as Teste de Valéry, Plume de Michaux, Crabe de Chevillard or Onze de Ledurt. Here munste appears as a little girl in the poetry of her childhood, in the sense that all childhood is a lost poem

munste n'a pas l'âge des adultes morts qui croient à l'enfance lavée dans le propre

munste se coule en ses rêves sans navire puis marche et déborde sur la rivière joue dans son lit avec ses petits cailloux

entre ses ongles les tulipes s'éveillent à force de déchirer toute blessure munste se trouve une chair mal décousue qui ajoute à la dérive certains restes

nul quai pour l'abordable océan promis aux peaux caressées sans leur corps véritable ou enfermées au plus profond des clés creuses

munste lance tous ses cailloux aux reflets ce sont les vitres qui se brisent calmées à moins que des obscurités sans contour ne s'érigent en toiles pinceaux pigments

si munste avait mieux su se prendre au piège peut-être aurait-elle pu y échapper

à porter son nom munste deviendra folle complètement ancrée à sa hauteur telle qu'il lui faudra sauter sauter de très haut oublier la teneur des phrases parfaites

qui se charge de la suspension des choses suit des routes en fuite mal consolées si bien que jaillissent les vierges seules 
munste ira aux larmes vivre sans mourir voir si une mare existe au fond des lacs quand les miroirs penchent sans plus retourner

1'utopie d'une baignoire en son émail la souffle vers des couleurs si fendillées

munste a beau mentir personne ne la croit on la place ici comme un objet mobile on lui parle de temps en temps on la peint autour d'elle le centre du monde tombe dans l'oubli forcé des prisons arrêtées

elle imposerait des sangles au désert puis enfermerait tous les récalcitrants dans une aiguille jusqu'à la pauvreté serait-elle un peuple elle aurait une langue par habitude des écrans mal vidés

munste naît dans la neige avec l'infini de la pluie sur son enfance en éclats troubles puis frôle ses armes l'amer à la bouche chutant du délire avec exactitude elle se touche à trop attendre le gel

un coquillage un pétale un havre vide munste ramasse ses morceaux s'y mélange elle fabrique cette petite chose de la grandeur d'un bateau incomparable

la couleur du ciel quand la lumière éclate

munste ouvre le jaune en tube pour la lune de son dessin sans veine ni relief pourtant elle revient récolter le sable puis poursuivre les fruits le vent de la houle 
sous l'horizon sa chambre a une fenêtre elle contourne le beige et crie le nord ses mains s'envolent pour des jeux volontaires

forcée à grandir malade en quarantaine munste se pose une question qu'elle tait presque aussi douce que la vitre brisée d'où les autres la regardent sans savoir

munste monte au dernier étage du rouge il n'y a que sa mère morte et la pluie

elle existe dans l'effusion du silence

entre les cadres une trace s'étend s'exténue en musique sans plénitude quand munste devient sa décision finale

sans tout ce qui ne laisse pas de séquelles munste se persuaderait du contraire

guérir par mascarade sur une plage accompagnée par la surface séchée avoir l'impression de vivre pour chanter

ses yeux clos se limitent à la douleur

munste se marierait sous une banquise rendrait son anémie par pleines poignées

un abat-jour éclaire les murs brûlés quand munste gémira l'ordre de ses gestes le passé sera convenu par écrit ainsi ses incontournables dégelées

munste esquisse une cale où des rats sans yeux s'effraient lorsqu'on les grève de la rougeur elle mouille ses lèvres de cécité arrive à peine à défendre la lumière

l'entrée se trouve quand on cherche à sortir aussi munste flanche lorsqu'elle est lancée

sa pudeur d'argile recouvre ses formes sous trois couches de peintures écaillées 